Elsevier

BBA 73674

\title{
Lipid specific penetration of melittin into phospholipid model membranes
}

\author{
A.M. Batenburg ${ }^{\text {a }}$, J.C.L. Hibbeln ${ }^{a}$ and B. de Kruijff ${ }^{b}$ \\ ${ }^{a}$ Department of Biochemistry and ${ }^{b}$ Institute of Molecular Biology and Medical Biotechnology, State University of Utrecht, \\ Utrecht (The Netherlands)
}

(Received 6 March 1987)

Key words: Melittin; Protein-lipid interaction; Fluorescence quenching; Melittin purification; Cardiolipin; Phosphatidylcholine; Model membrane; (Bovine heart)

The relative depth of penetration of melittin into egg phosphatidylcholine and bovine heart cardiolipin model membranes was investigated using fluorescence spectroscopy techniques. The tryptophan intrinsic fluorescence shift suggests a more hydrophobic surrounding of this residue in cardiolipin, while the accessibility for charged and uncharged aqueous quenchers is decreased in the cardiolipin system when compared with the phosphatidylcholine-bound situation. A lipid incorporated hydrophobic, collisional quencher and a resonance energy transfer acceptor on the other hand are more effective in quenching the tryptophan fluorescence of cardiolipin bound melittin. The combination of these results is interpreted as prove of a deeper positioning of the tryptophan containing part of the peptide molecule in the cardiolipin system in comparison with the situation in phosphatidylcholine. Models that take this difference into account are presented, which try to explain the opposite effect of melittin binding to the two lipid systems with respect to supramolecular structure, as reported in the preceding article (Batenburg, A.M., Hibbeln, J.C.L., Verkleij, A.J. and De Kruijff, B. (1987) Biochim. Biophys. Acta 903, 142-154).

\section{Introduction}

The amphiphilic bee venom component melittin strongly interacts with biological membranes and pure lipid bilayers. The position of melittin with respect to the phospholipids to which it is bound, as well as its aggregational state in this situation have been subject of several studies [2-19] and considerable debate. The vast majority of these reports were limited to the insertion of melittin into detergent micelles or phosphati-

Abbreviations: PC, phosphatidylcholine; SUV, small unilamellar vesicles.

Correspondence: A.M. Batenburg, Department of Biochemistry, State University of Utrecht, P.O. Box 80.054, 3508 TA Utrecht, The Netherlands. dylcholine bilayers. Our ${ }^{31} \mathrm{P}-\mathrm{NMR}$, freeze-fracture and $\mathrm{X}$-ray diffraction studies however, as reported in the preceding article [1] show a remarkable difference between the structural alterations induced by melittin in the negatively charged cardiolipin system (formation of inverted non-bilayer structures) and those reported for phosphatidylcholines (formation of disc and micellar type of structures $[16,19])$. The hypothesis that this lipid specific structural reorganization is correlated with a different mode of insertion is addressed in this report, using fluorescence techniques. The data obtained indeed all point to a deeper penetration of the sequence around tryptophan-19 into the lipid core in the cardiolipin system when compared with insertion into phosphatidylcholine bilayers. These results are integrated in a model of melittin induced changes in lipid organization. 
One of the biological activities of melittin is its promotion of the action of phospholipase $\mathrm{A}_{2}$ $[20,21]$ which is present in all commercial samples of melittin. If not removed, this enzyme will cause rapid hydrolysis of phospholipids that can lead to erroneous results in studies of melittin-membrane interaction, as established by Dasseux et al. [22]. Since all reported purification procedures were found unsatisfactory, we also developed a simple and mild procedure, with a recovery of $80-90 \%$, for the complete removal of phospholipase traces from melittin, based on covalent chromatography.

\section{Materials and Methods}

\section{Materials}

Commercial samples of melittin were purchased from Serva (Heidelberg, F.R.G.) (reinst. contr. nr. $\mathrm{H}$ and pharm. lot nr. 18015) and Sigma (St. Louis, U.S.A.) (lot 12 F-0078). Apis mellifera venom was obtained from Serva and Sigma. Sephadex G-25 and Thiopropyl-Sepharose 6B (2-pyridyldisulphidehydroxypropyl-ether-sepharose) were from Pharmacia (Uppsala, Sweden). Egg yolk PC (fatty acid composition: $33 \% 16: 0 ; 29 \% 18: 1$; $18 \% 18: 2 ; 16 \% 18: 0 ; 2 \% 16: 1$ and $2 \% 20: 4)$ and soy bean PC (fatty acid composition: $60 \% 18: 2$, $20 \% 16: 0 ; 12.5 \% 18: 1 ; 4 \% 18: 0$ and $2.5 \% 18: 3$ ) were isolated according to standard procedures and dioleoyl-PC was synthesized from egg yolk $\mathrm{PC}$ as described before [23]. L- $\alpha$-Di[ $1-{ }^{14}$ C $]$ oleoylPC was from New England Nuclear (Dreieich, F.R.G.) (NEC 682, 80-120 $\mathrm{mCi} / \mathrm{mmol}$ ). Cardiolipin from bovine heart (composition: $80 \%$ $18: 2 ; 7.5 \% 18: 3 ; 6 \% 18: 1 ; 4 \% 16: 1 ; 1 \% 18: 0$; $1 \% 20: 4$ and $0.5 \% 16: 0$ ) was purified according to Smaal et al. [24] and petroselinic acid (1-octadeca-6-enoic acid) was from $\mathrm{Nu}$-Chek-Prep, Inc. (Elysian, MI, U.S.A.).

\section{Melittin purification}

$50 \mathrm{mg}$ of a partly purified melittin sample (Serva, pharm. grade, 18015) were dissolved in 250 $\mu 10.3 \mathrm{M} \mathrm{NaHCO}, 1 \mathrm{mM}$ EDTA (pH 8.4) to which $1 \%$ dithiothreitol $(\mathrm{w} / \mathrm{v})$ was added. During an incubation at room temperature for $40 \mathrm{~min} \mathrm{a}$ precipitate was formed which was pelleted by a 15 s $5000 \times \mathrm{g}$ centrifugation. Excess reductor was removed by gelfiltration in a $1 \times 40 \mathrm{~cm}$ column of
Sephadex G-25, eluted with $0.01 \mathrm{M}$ sodium acetate buffer ( $\mathrm{pH} \mathrm{4.0)}$ to prevent reformation of sulphur bridges. The effluent fractions containing the peptide were collected in $5 \mathrm{ml} 0.1 \mathrm{M}$ Tris- $\mathrm{HCl}, 1$ mM EDTA ( $\mathrm{pH} 7.5$ ) containing $250 \mathrm{mg}$ Thiopropyl-Sepharose $6 \mathrm{~B}$ which had been swollen and washed with water according to the supplier's instructions. An incubation for $20 \mathrm{~min}$ at room temperature was followed by removal of the agarose beads on a glass-filter and incubation for another $20 \mathrm{~min}$ with second amount of $250 \mathrm{mg}$ Thiopropyl-Sepharose. Without concentration the supernatant was loaded on a $2.2 \times 25 \mathrm{~cm}$ HPLC column filled with Si $100(10 \mu \mathrm{m})$ Polyol RP-18 (Serva) and equipped with a Gilson model 303 pump and a Knauer 8700 variable wavelength detector set at $280 \mathrm{~nm}$. The column was eluted at a flow of $10 \mathrm{ml} / \mathrm{min}$ with a linear gradient of $70-95 \%$ methanol in water containing $0.5 \%$ trifluoroacetic acid. Melittin was collected at 83-88\% methanol and was lyophilized after addition of 2 vol. of water.

\section{Analyses of melittin samples}

Phospholipase $\mathrm{A}_{2}$ contamination in the melittin samples was determined by incubation of an unsonicated dispersion of ${ }^{14} \mathrm{C}$-labeled dioleoyl-PC $(0.6 \mu \mathrm{mol})$ with $0.1 \mathrm{mg}$ of the melittin sample to be tested in $10 \mu \mathrm{l} 10 \mathrm{mM}$ Tris- $\mathrm{HCl}, 100 \mathrm{mM}$ $\mathrm{NaCl}, 10 \mathrm{mM} \mathrm{CaCl}{ }_{2}(\mathrm{pH} 7.0)$ for $1 \mathrm{~h}$ at $37^{\circ} \mathrm{C}$, after which phospholipid and degradation products were separated on HPTLC plates (Merck 11845 ) and their amounts determined by scintillation counting.

For amino acid analysis samples were analysed on a Kontron Labotron Pro 1 after hydrolysis in 6 $\mathrm{M} \mathrm{HCl}$ for $20 \mathrm{~h}$ at $110^{\circ} \mathrm{C}$ in vacuum. For analytical HPLC melittin fractions ( $100 \mu \mathrm{g}$ in $10 \mu \mathrm{l} \mathrm{H}_{2} \mathrm{O}$ ) were separated on a $0.45 \times 25 \mathrm{~cm}$ column in the experimental set-up mentioned above and containing the same reversed phase material. The column was eluted with $1 \mathrm{M} \mathrm{LiClO}_{4}(\mathrm{pH} \mathrm{2.1)}$ at a flow rate of $1 \mathrm{ml} / \mathrm{min}$. A linear gradient of $0-90 \%$ acetonitrile was applied at a rate of $2 \% / \mathrm{min}$. The elution profile at $220 \mathrm{~nm}$ was recorded.

\section{Model membranes}

From phospholipid dispersions, made by hydration of a dry lipid film, small unilamellar 
vesicles (SUV) were prepared by a $10 \times 30$ s $50 \mathrm{~W}$ sonication under nitrogen atmosphere in ice-water, with a Branson B12 sonifier equipped with a 0.5 -inch flat-top disruptor and isolated as the supernatant of a 15 -min $27000 \times g$ centrifugation. Vesicles were prepared and all experiments performed in $100 \mathrm{mM} \mathrm{NaCl}, 10 \mathrm{mM}$ Tris- $\mathrm{HCl}$ and 1 $\mathrm{mM}$ EDTA $(\mathrm{pH} 7.0)$ at $25^{\circ} \mathrm{C}$, unless otherwise stated. Phospholipid concentration was determined by a phosphorus assay after destruction with perchloric acid $[25,26]$.

\section{Intrinsic fluorescence measurements}

Changes of tryptophan intrinsic fluorescence upon lipid binding were measured by titration of a $10 \mu \mathrm{M}$ melittin solution with phospholipid SUV. Two minutes after each addition an emission spectrum was recorded with a Perkin-Elmer MPF3 fluorometer, the excitation wavelength being 280 $\mathrm{nm}$. After correction for volume change, the percentual increase of fluorescence intensity at a fixed wavelength was calculated. The melittin-induced time-dependent increase of light scattering had less than $5 \%$ influence on the measured emission as judged from measurements at wavelengths where no fluorescence occurs (excitation $340 \mathrm{~nm}$, emission $400 \mathrm{~nm}$ ). In order to analyse the data in terms of a dissociation constant $\left(K_{\mathrm{d}}\right)$ and a number of lipid monomers per peptide binding site $(N)$ a nonlinear regression method [27] was applied based on the equation $K_{\mathrm{d}}=[\mathrm{P}]\left[\mathrm{L}_{\mathrm{N}}\right]\left[\mathrm{PL}_{\mathrm{N}}\right]^{-1}$ in which $[\mathrm{P}]$ and $\left[\mathrm{L}_{\mathrm{N}}\right]$ are the concentrations of free peptide and free peptide binding sites, respectively.

\section{Measurements of the influence of aqueous quenchers}

The ability of polar solutes to quench the tryptophan fluorescence was studied by addition of increasing amounts of a $2 \mathrm{M}$ sodium nitrate, a 3 $\mathrm{M}$ acrylamide or a $4 \mathrm{M}$ potassium iodide solution to $10 \mu \mathrm{M}$ melittin. The iodide solution additionally contained $1 \mathrm{mM} \mathrm{Na}{ }_{2} \mathrm{~S}_{2} \mathrm{O}_{3}$ to prevent $\mathrm{I}_{3}^{-}$formation. If the effect of quenchers in the presence of lipid was studied, titration was started $2 \mathrm{~min}$ after mixing of melittin with SUV. The contribution of light scattering caused by the vesicles, which was not significantly increased by melittin in the peptide-lipid ratio used, was subtracted. The results were incorporated in Stern-Volmer plots, from which the dynamic quenching constants, correlating with the slopes, could be determined with an accuracy of about $5 \%$.

Fluorescence quenching experiments with brominated fatty acids

6,7-Dibromostearic acid was prepared by addition of a 2-fold excess of $\mathrm{Br}_{2}$ solution in $2 \mathrm{ml}$ chloroform to $30 \mathrm{mg}$ petroselinic acid. After a one hour incubation at $-20^{\circ} \mathrm{C}$, excess bromine was removed by extraction with $3 \mathrm{ml}$ concentrated $\mathrm{Na}_{2} \mathrm{~S}_{2} \mathrm{O}_{3}$ solution and washing with $1 \mathrm{ml}$ of water. Gas chromatography after methylation of the fatty acids, using heptadecanoic acid as external standard, evidenced the remains of less than $3 \%$ of the starting material. The brominated fatty acid was added in different amounts to the phospholipid solution prior to the formation of the dry lipid films, since external addition to the SUV resulted in irreproducible results.

\section{Tryptophan to anthracene resonance energy transfer measurements}

The overlap of the tryptophan fluorescence and the anthracene excitation spectrum makes it possible to study the depth of insertion of a tryptophan containing peptide into a membrane via measurement of energy transfer to membrane embedded anthracene [28,29]. Anthracene was added as a 1 $\mathrm{mM}$ ethanolic solution to the SUV; if melittin was present, addition was started 2 min after mixing of lipid and peptide. After another $2 \mathrm{~min}$, necessary for a complete stabilization of the spectrum, the emission was recorded and was found to be similar to the spectrum obtained from vesicles in which anthracene was incorporated by addition to the lipid solution in organic solvent, prior to preparation of the dry film.

\section{Results}

\section{Melittin purification}

All melittin samples obtained from commercial suppliers contained considerable amounts of phospholipase $\mathrm{A}_{2}$ (30 to more than $100 \mathrm{mIU} / \mathrm{mg}$ ), which appeared to be only partially blocked by 1 mM EDTA in the absence of $\mathrm{CaCl}_{2}$ under the conditions of our phospholipase assay. Several methods to purify melittin from commercial sam- 
ples as well as from whole bee venom were tested. Hydrophobic interaction chromatography on Phenyl-Sepharose CL-4B and reversed phase HPLC with $5 \%$ formic acid and a gradient of isopropanol appeared to be uneffective in our hands as did gel-filtration through Sephadex G-100 [7]. Melittin without detectable phospholipase contamination could, however, be obtained both from crude melittin samples and from whole bee venom (data not shown) by reduction of the disulphide bonds of the phospholipase, followed by extraction of melittin with butanol [21]. Our yields were always low ( $<30 \%$ melittin recovery), due to the formation of a sticky precipitate, as was reported by others [30]. Replacement of the butanol extraction by precipitation of the contaminants with an excess of $n$-propanol gave high yields ( $>80 \%$ ); the product, however, was contaminated with a substantial amount of phospholipase.

Removal of the reduced phospholipase from crude melittin samples by covalent binding to Thiopropyl-Sepharose 6B resulted, after desalting through Sephadex G-25, in a melittin preparation devoid of any measurable phospholipase activity, even when tested in prolonged incubations for $5 \mathrm{~h}$. Melittin could be desalted and separated from some other minor contaminants, that did not bind to Thiopropyl-Sepharose, by gradient elution through a preparative reversed phase HPLC column (Fig. 1). Most of the impurities, constituting about $5 \%$ of the eluted dry mass (fraction II), were recovered from the column shortly after the melittin peak (fraction I). The entire procedure yielded $38-43 \mathrm{mg}$ of melittin from $50 \mathrm{mg}$ of a crude commercial melittin sample.

The amino acid analysis of the purified melittin correlated well with the expected values [31]. HPLC analysis, with detection at $220 \mathrm{~nm}$ to monitor also possible nonaromatic contaminants, showed a single peak eluting at $62 \%$ acetonitril (Fig. 2A). The contaminant fraction II contained some alien amino acid species, though had roughly the same composition as melittin, suggesting a domination of this fraction by $N^{\alpha}$-formylmelittin, a minor venom component [32], which on basis of its slightly increased hydrophobicity may be expected to elute at somewhat higher concentration of organic solvent in HPLC, as was indeed observed (Figs. 1 and 2).

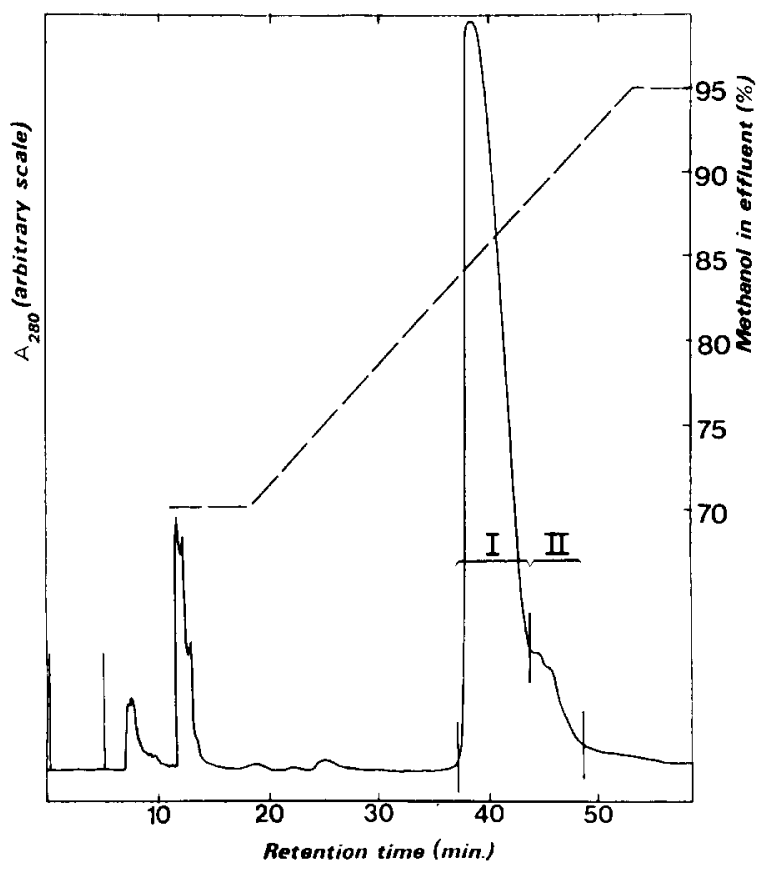

Fig. 1. Preparative HPLC of a crude melittin preparation (Serva, lot 18015) after treatment with Thiopropyl-Sepharose 6B. The sample was applied to the column from $t=0$ to $t=5$ $\min$.

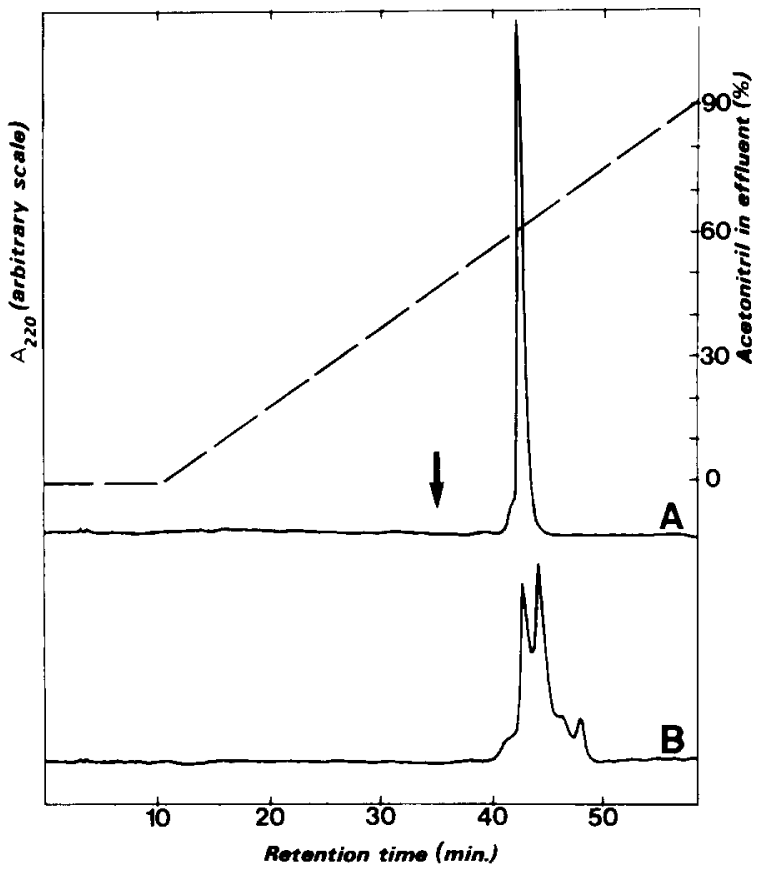

Fig. 2. HPLC analyses of the fractions I and II (A and B, respectively) from the preparative HPLC run shown in Fig. 1. The arrow indicates the position where bee venom phospholipase would elute if present. 


\section{Intrinsic fluorescence}

To obtain a first insight in the penetration of melittin into cardiolipin model membranes the change of the tryptophan-19 intrinsic fluorescence itself upon cardiolipin binding was studied. As shown by Fig. 3, binding results in a $20 \mathrm{~nm}$ blue shift and increased intensity of the emission, both indicative of a more hydrophobic environment, most likely formed by the apolar parts of the lipids. Binding to egg-PC vesicles effects a shift of only 16 to $17 \mathrm{~nm}$ to a final value of $335 \mathrm{~nm}$ (data not shown) in agreement with the average of the data reported earlier $[2,6,7]$. This difference is a first indication suggestive of a deeper penetration in the cardiolipin system, though it should be kept in mind that depth of penetration and blue shift of fluorescence are not directly related. The fluorescence changes reach a plateau value at a phospholipid to melittin ratio of $2.03 \pm 0.07: 1$, which is in good agreement with the stoichiometry established with a centrifugation based binding assay [1]. The $K_{\mathrm{d}}$ was calculated to be $(1.6 \pm 0.7)$. $10^{-8} \mathrm{M}$.

\section{Influence of aqueous quenchers}

More direct information about the localization of the tryptophan can be obtained from its accessibility towards aqueous quenchers. Fig. $4 \mathrm{~A}$ indicates that the fluorophore is almost completely shielded from the aqueous quencher $\mathrm{I}^{-}$in both lipid systems. The observed difference in quenching efficiency, however, suggests a yet even better

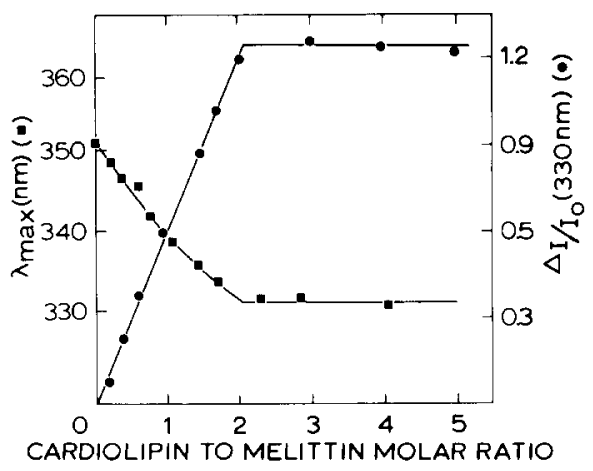

Fig. 3. Effect of binding of melittin to cardiolipin SUV on tryptophan fluorescence intensity (e) and wavelength of maximal fluorescence (a). Experiments were performed as described under Materials and Methods. $I_{0}$ is the initial emission intensity, $\Delta I$ is the increase of intensity upon lipid addition. protection when melittin is bound to cardiolipin.

Quenching with $\mathrm{NO}_{3}^{-}$offers more discriminative power (Fig. 4B) since this ion apparently has more penetrative capacity. The quenching constants, $K_{\mathrm{SV}}$, for the peptide free in solution and when bound to egg-PC, of $55 \mathrm{M}^{-1}$ and $12.8 \mathrm{M}^{-1}$,
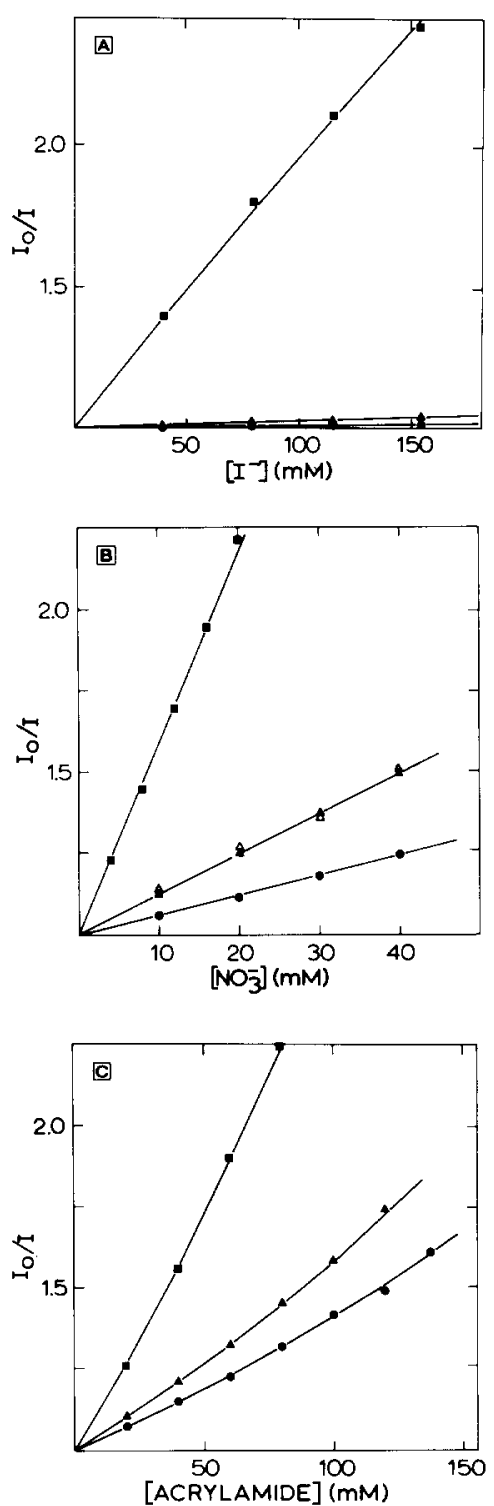

Fig. 4. Stern-Volmer plots of the quenching of melittin intrinsic fluorescence by iodide (A); nitrate (B) and acrylamide (C) in the absence of lipid $(\square)$ and in the presence of either soybean PC $(\Delta)$, egg PC $(\Delta)$ or cardiolipin (๑). Initial concentrations: melittin, $5 \mu \mathrm{M}$; phospholipid $0.5 \mathrm{mM}$ (based on lipid phosphorus); $\lambda_{\mathrm{ex}}=280 \mathrm{~nm}, \lambda_{\mathrm{em}}=330 \mathrm{~nm}$. 
respectively, well agree with previously reported data $\left(51.3 \mathrm{M}^{-1}\right.$ and $13.8 \mathrm{M}^{-1}$ for free and DMPC-bound melittin [7]). In cardiolipin complexes a much more pronounced decrease of the quenching constant to a value of $6.4 \mathrm{M}^{-1}$ is observed. Assuming no major differences in fluorescence decay time $\tau$ between the studied situations, as justified by earlier reports [7], this points to a better shielding of the indole group of tryptophan-19 towards $\mathrm{NO}_{3}^{-}$in the cardiolipin system when compared with egg PC. The effect is not caused by differences in acyl-chain composition, since soybean PC, with an unsaturation much closer to that of bovine heart cardiolipin, shows a protection of the tryptophan similar to that of egg PC (Fig. 4B).

The improved shielding by cardiolipin is not limited to charged quenchers, as evidenced by Fig. $4 \mathrm{C}$, where the effect of acrylamide titration on the fluorescence of melittin in different environments is shown. The difference in accessibility in the two lipid systems is not as distinct as for $\mathrm{NO}_{3}^{-}$, which might, at least partially, be explained by the notion that acrylamide partitions into the interior of the membrane [33]. The collisional quenching constants were estimated to be $12.5 \mathrm{M}^{-1}, 3.8 \mathrm{M}^{-1}$

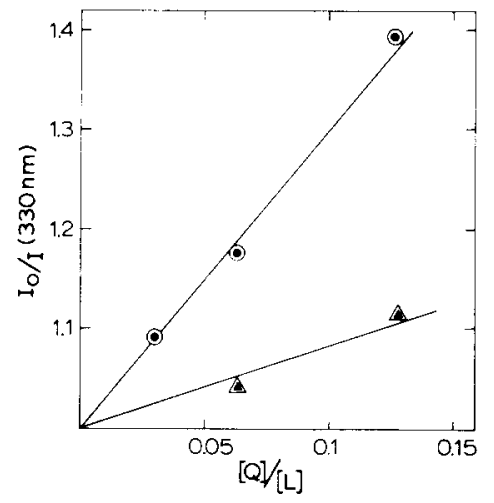

Fig. 5. Quenching of melittin tryptophan fluorescence by 6,7dibromostearic acid incorporated in egg-PC $(\boldsymbol{\Delta})$ or cardiolipin (-) membranes. The surface concentration of the quencher is expressed as its molar ratio to lipid phosphorus. Excitation wavelength $280 \mathrm{~nm}$, emission wavelength $335 \mathrm{~nm}$; concentrations: $2 \mu \mathrm{M}$ melittin, $200 \mu \mathrm{M}$ lipid phosphorus.

and $2.6 \mathrm{M}^{-1}$ for the peptide in aqueous solution and when bound to egg $\mathrm{PC}$ and cardiolipin, respectively. For the free peptide this is in good agreement with published data, but for quenching of egg PC-bound melittin our value is considerably higher than reported before [13]; we have no explanation for this discrepancy.
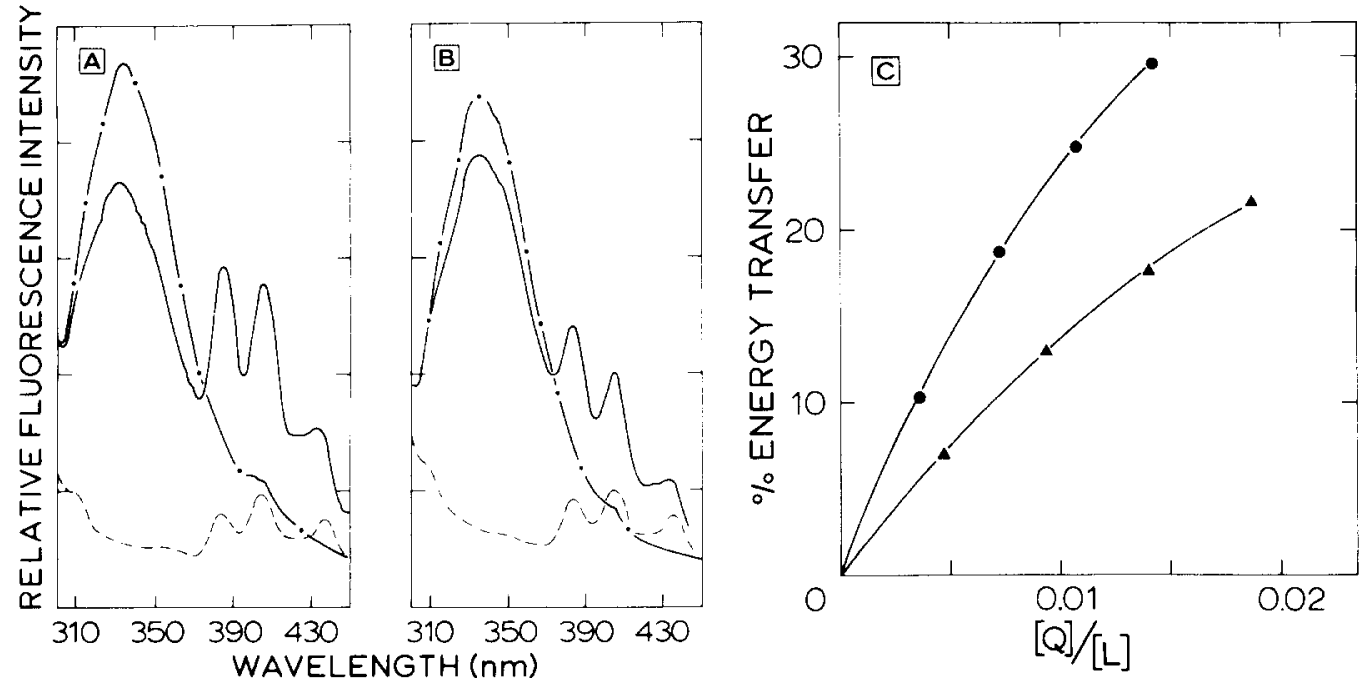

Fig. 6. Energy transfer from melittin tryptophan to membrane incorporated anthracene. In (A) and (B) the emission spectrum of melittin is shown when bound to cardiolipin (A) and egg PC (B) in the absence $\left(\cdot \cdot_{-} \cdot\right)$ ) and presence $(-\longrightarrow)$ of anthracene, added in a molar ratio of 1:80 to lipid phosphorus; the emission of this amount of anthracene in the absence of melittin is also shown ( - - - . In (C) the energy transfer efficiency as calculated from the effect of anthracene titration on the $335 \mathrm{~nm}$ emission of melittin, bound to cardiolipin (๑) or egg PC ( $\triangle)$ is depicted. Quencher concentration is expressed as molar ratio to lipid phosphorus. The excitation wavelength was $280 \mathrm{~nm}$, the melittin concentration $3.0 \mu \mathrm{M}$ and the lipid concentration $175 \mu \mathrm{M} \mathrm{P}_{\mathrm{i}}$. 


\section{Influence of hydrophobic quenchers}

As indicated by the about 4-fold larger quenching efficiency, the accessibility of melittin's tryptophan-19 towards a lipid incorporated collissional quencher, 6,7-dibromostearic acid, appeared to be clearly enhanced in the cardiolipin bound situation when compared with that in egg PC (Fig. 5), again assuming no difference in excited state lifetime. This is interpreted as further evidence for a deeper penetration of the (tryptophan containing part of the) peptide into the cardiolipin core. Incorporation of similar amounts of stearic acid did not change the fluorescence intensity of egg PC-bound melittin, indicating that the low quenching efficiency was not effected by compensatory fluorescence increase resulting from the introduction of negative charges.

The characterization of melittin penetration in egg-PC and cardiolipin membranes was completed with a study of the tryptophan fluorescence quenching by anthracene (Fig. 6), which on basis of its hydrophobicity may safely be expected to be located in the acylchain region. Quenching in this case is based on resonance energy transfer from tryptophan to anthracene, which results in a concomitant increase of the anthracene emission around $400 \mathrm{~nm}$ as shown by Fig. 6A and 6B. Since the same fixed anthracene to lipid phosphorus ratio was applied in these experiments, the two plots can directly be compared and plainly show a more efficient energy transfer in the cardiolipin system than in the egg-PC membranes. The results of the titration experiments (Fig. 6C) imply that a twice as large anthracene concentration is needed in egg PC when compared with cardiolipin, to obtain a similar efficiency of energy transfer, thus again indicating a deeper insertion into the cardiolipin membrane.

\section{Discussion}

Melittin was freed from phospholipase $\mathrm{A}_{2}$ with a newly developed procedure having distinct advantages over previously published ones with respect to simplicity, mildness, recovery and effectiveness. With this thoroughly purified material the penetration of melittin into PC and cardiolipin was investigated in a comparative way, in order to explain the completely opposite effect of melittin on the macroscopic organization of these two phospholipids [1]. The mode of insertion of melittin into zwitterionic phospholipids has been extensively investigated already with many techniques. Free melittin is known to form tetramers at high concentration and high ionic strength [34]; recent fluorescence energy transfer measurements, though in principle very elegant, have led to exactly opposite conclusions $[17,18]$ with respect to the possible multimeric state of membrane-bound melittin. From earlier ${ }^{1} \mathrm{H}-\mathrm{NMR}$ data $[4,5]$, however, evidence for binding as monomers was inferred.

The other controversies too, regarding the orientation and depth of penetration of melittin in the lipid matrix, are far from being settled. On basis of the expected amphipathicity $[3,8]$ of the helices that are formed during binding to phospholipids $[7,35]$ some authors $[8,3]$ have proposed that the orientation of the melittin molecule is parallel to the membrane surface, perhaps with the exception of the smaller C-terminal helix 15-21 [3]. Polarized infrared spectroscopy studies [15], however, have provided experimental evidence in favour of a mean orientation of the helices perpendicular to the plane of the bilayer, as a result of either a transmembrane orientation as suggested earlier [9] or a 'loop'-orientation with $\mathrm{C}$ and $\mathrm{N}$-terminus at the same side of the lipid layer involving a sharp nick around residue 13 , a model supported also by others [10] on basis of proton NMR studies.

Concerning the depth of insertion, ${ }^{13} \mathrm{C}-\mathrm{NMR}$ data [14] and photo-labelling results [36] suggested a deep penetration of the peptide, but from ${ }^{1} \mathrm{H}$ NMR data in micelles a penetration to a depth of only $6-8 \AA$ was inferred [10]. From fluorescence [2] and ${ }^{1} \mathrm{H}-\mathrm{NMR}$ [4] data a deep penetration of the tryptophan containing part of the peptide was concluded; more recent experiments with fluorescence quenchers $[7,13]$, however, indicated a more superficial position of the Trp-19, most likely in the glycerol backbone area [7]. Whatever the reasons for these discrepancies, most of them probably being related to the differences in techniques and model systems used, in the present study the penetration in two lipid systems is examined in a comparative, rather than an absolute way.

The results presented in this report point to a 
lipid specificity of the penetration of melittin into phospholipid systems. The fluorescence maximum at $331 \mathrm{~nm}$ of cardiolipin-bound melittin suggests a surrounding of lower dielectric constant of the tryptophan residue when compared with the egg PC-bound situation where fluorescence is centered around $335 \mathrm{~nm}$. The further decreased accessibility towards aqueous quenchers confirms this deeper penetration in the cardiolipin system. For the quenchers iodide and nitrate the lower quenching efficiency may partly be due to the repulsing charges of quencher and lipid, yet for the neutral acrylamide this is not the case.

More evidence for a different localization of the fluorophore in the two lipids, complementary to the results with the aqueous quenchers, was inferred from the effectivity of the hydrophobic, membrane incorporated 6,7-dibromostearic acid and anthracene to quench the tryptophan emission. When compared with the maximal efficiency of brominated lipids to quench the tryptophan of lipid-bound pancreatic phospholipase [37], the quenching by the brominated fatty acid suggests a tryptophan position close to the bromine atoms when melittin is inserted in cardiolipin.

The energy transfer efficiency from the melittin tryptophan to anthracene is in PC about $75 \%$ of what can be expected according to the theoretical curves of Fung and Stryer [38] in case of a location of donor and acceptor at a similar depth, at a position where transfer to acceptors in the other monolayer is negligible $\left(R_{0}\right.$, the distance at which transfer rate equals donor decay rate, is approx. $20 \AA$ [39]). This could be in agreement with a location of Trp-19 in the glycerol backbone area [7]. The efficiency in cardiolipin on the other hand, being twice as large, points to a position more close to the centre of the hydrophobic core. Application of Försters equation for efficiency of energy transfer $E=R^{-6} /\left(R^{-6}-R_{0}^{-6}\right)$ leads to a $7 \AA$ deeper insertion of the indole group in cardiolipin, assuming in first approximation all anthracene molecules concentrated at the bilayer midplane and the melittin tryptophans in PC positioned at $15 \AA$ away from this midplane. From other approximations, involving distribution of anthracene over the hydrophobic core of the membrane, an $8-10 \AA$ deeper penetration in the cardiolipin system can similarly be inferred. Given however the incertainties in the parameters involved, for instance the exact acceptor location, the dipole-dipole orientation factor (here assumed to be $2 / 3$ ) and the effect of possible differences in bilayer packing and thickness, these values can only be taken as a rough estimate.

Nevertheless the clear difference in energy transfer efficiency in the two lipid systems, taken together with the results obtained with the hydrophobic and aqueous collisional quenchers, strongly points to a deeper positioning of the tryptophan containing part of melittin in bovine heart cardiolipin, when compared with egg PC. As indicated by nitrate quenching of soy bean PC-bound melittin, this difference does not correlate with the degree of acyl-chain unsaturation but rather originates from differences in headgroup properties.

In order to explain the different structures of PC-melittin and cardiolipin-melittin complexes [1], we will now propose models which take into account the different position of the tryptophan residue in these two lipid systems. For these models arbitrarily a monomeric and lipid independent structure of the peptide molecule is assumed and in the analysis different proposed orientations of the melittin helices will be considered. In an orientation of melittin parallel to the original bilayer plane, attraction of the negative charge of the cardiolipin headgroup by positive charges at the centre of the polar half of the amphipathic helix (Lys-7 and Lys-21) would force the peptide deeper into the lipid (Fig. 7 A1). The electrostatic interaction would also decrease interheadgroup repulsion and thereby headgroup size, which together with the action of the helix as acylchain spacer in this situation, could induce according to the shapestructure concept of the lipid polymorphism [40], the curvature typical for the type II (inverted hexagonal or inverted micellar) structures (Fig. 7 A1), that are actually formed in this complex. As indicated by the increased interaction at high salt concentration [41], binding to $\mathrm{PC}$ is governed by hydrophobic interaction, leading to an embedding of the hydrophobic side of the helices only, and hence a headgroup spacing effect. This 'wedge' effect, as suggested by others [3], would then induce the formation of the reported $[16,19]$ type I (micellar) structures (Fig. 7 A2). If, alternatively, a 

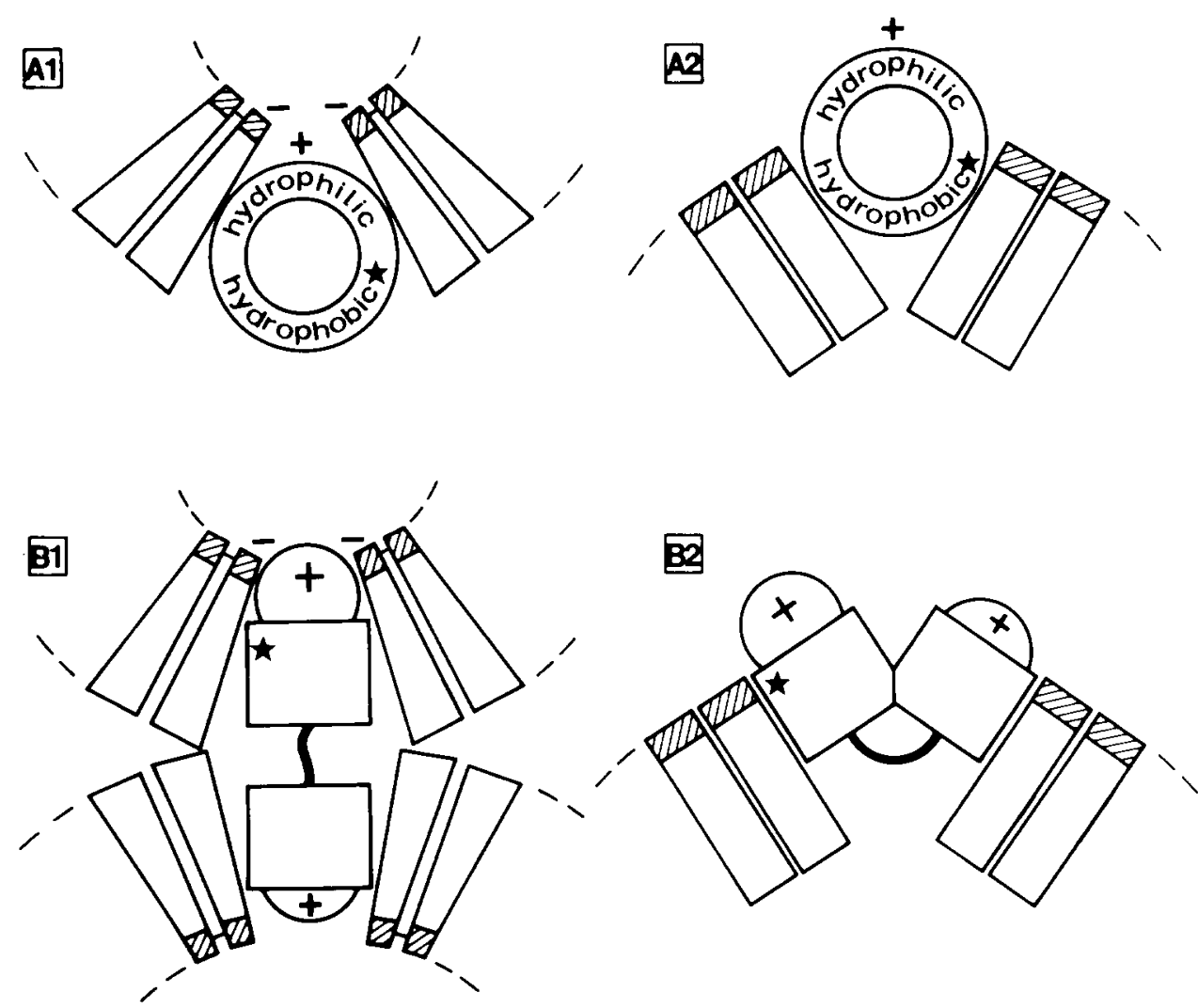

Fig. 7. Rationale for the lipid specificity of melittin-induced changes in phospholipid organization. Two models are presented, based on an alignment of the melittin helix either parallel (A) or perpendicular (B) to the original membrane plane. (A) shows cross-sectional views of the lipid inserted melittin helices, whereas in (B) side-views are depicted, the 'hinge' region, for which no helix is predicted, drawn as a thin flexible line. The position of the tryptophanyl residue at position 19 is indicated with a star. In (A) the location of Lys-7 and Lys-21 is indicated with a plus-sign, the other charges in this model being situated at undefined locations; in (B) the plus-signs reflect the charged residues at the extrema of the molecule, Lys-7 in these situations perhaps being deprotonated at the expense of interaction energy.

perpendicular orientation is assumed (Fig. 7 B) the formation of type II structures in cardiolipin is most easily conceived as the result of a transbilayer insertion of the melittin molecule, allowing full contact of opposite charges. The effect of this electrostatic interaction on interheadgroup repulsion and headgroup size, combined with the bulkiness of the helical region of the molecule and perhaps the mismatch of bilayer thickness and distance between $\mathrm{N}$ - and $\mathrm{C}$-terminal charges would then be the basis of the structural alterations (Fig. $7 \mathrm{~B} 1$ ). For the PC bound situation a bent rod conformation (Fig. 7 B2) would be in better agreement with the apparent position of the tryptophan; the wedge effect of such a mode of insertion could, here again, bring about the convex curvature of micellar structures. The knowledge gathered so far is not sufficient to make a well founded choice between these models and even combinations of for instance Fig. 7 A2 and B1 may better describe the actual situations. In a possible multimeric configuration of melittin in PC, a nicked transbilayer orientation (Fig. 10 of ref. 18) could well explain the micelle formation.

Interestingly, transitions between the different situations, which have been used earlier to explain voltage-dependent protease accessibility of melittin [12], could be of physiological importance. Melittin for instance bears distinct resemblance to the signal peptides of membrane and secretion proteins $[42,43]$ and it could be imagined that a peptide induced phase segregation, resulting in a surrounding enriched in negatively charged phospholipids, could lead to a transition of the orienta- 
tion of a membrane bound signal sequence from situation $\mathrm{A} 2$ or $\mathrm{B} 2$ (Fig. 7) to situation $\mathrm{B} 1$ and thus to translocation of the site were the mature sequence of the protein is attached, the mentioned situations being sequential steps in the export process. Many export-models are based on signal peptide-phospholipid interaction [44-46] and it would be worthwile to investigate further the role of these orientations and their consequences for phospholipid structure [1] using melittin as a model peptide or, better, synthetically prepared signal sequences.

\section{Acknowledgements}

We thank W. Atsma for performing the amino acid analyses and Dr. C. Mollay for advice in the initial stages of the development of the purification procedure. This work was carried out under the auspices of the Netherlands Foundation of Chemical Research (S.O.N.) and with financial aid from the Netherlands Organization for the advancement of Pure Research (Z.W.O.).

\section{References}

1 Batenburg, A.M., Hibbeln, J.C.L., Verkleij, A.J. and De Kruijff, B. (1987) Biochim. Biophys. Acta 903, 142-154

2 Dufourcq, J. and Faucon, J.-F. (1977) Biochim. Biophys. Acta 467, 1-11

3 Dawson, C.R., Drake, A.F., Helliwell, J. and Hider, R.C. (1978) Biochim. Biophys. Acta 510, 75-86

4 De Bony, J., Dufourcq, J. and Clin, B. (1979) Biochim. Biophys. Acta 552, 531-534

5 Lauterwein, J., Bosch, C., Brown, L.R. and Wüthrich, K. (1979) Biochim. Biophys. Acta 556, 244-264

6 Georghiou, S., Thompson, M. and Mukhopadhyay, A.K. (1981) Biochim. Biophys. Acta 642, 429-432

7 Vogel, H. (1981) FEBS Lett. 134, 37-42

8 Terwilliger, T.C., Weissman, L. and Eisenberg, D. (1982) Biophys. J. 37, 353-361

9 Lavialle, F., Levin, I.W. and Mollay, C. (1980) Biochim. Biophys. Acta 600, 62-71

10 Brown, L.R., Braun, W., Kumar, A. and Wüthrich, K. (1982) Biophys. J. 37, 319-328

11 De Grado, W.F., Musso, G.F., Lieber, M., Kaiser, E.T. and Kezdy, F.J. (1982) Biophys. J. 37, 329-338

12 Kempf, C., Clausner, R.D., Weinstein, J.N., Van Renswoude, J., Pincus, M. and Blumenthal, R. (1982) J. Biol. Chem. 257, 2469-2476

13 Georghiou, S., Thompson, M. and Mukhopadhyay, A.K. (1982) Biochim. Biophys. Acta 688, 441-452

14 Coddington, J.M., Johns, S.R., Willing, R.I., Kenrick, J.R. and Bishop, D.G. (1983) Biochim. Biophys. Acta 727, 1-6
15 Vogel, H. Jähnig, F., Hoffmann, V. and Stümpel, J. (1983) Biochim. Biophys. Acta 733, 201-209

16 Dufourcq, J., Faucon, J.-F., Fourche, G., Dasseux, J.L., Le Maire, M. and Gulik-Krzywicki, T. (1986) Biochim. Biophys. Acta $859,33-48$

17 Hermetter, A. and Lakowicz, J.R. (1986) J. Biol. Chem. 261, 8243-8248

18 Vogel, H. and Jähnig, F. (1986) Biophys. J. 50, 573-582

19 Dufourc, E.J., Faucon, J.-F., Fourche, G., Dufourcq, J., Gulik-Krzywicki, T. and Le Maire, M. (1986) FEBS Lett. 201, 205-209

20 Mollay, C. and Kreil, G. (1974) FEBS Lett. 46, 141-144

21 Mollay, C., Kreil, G. and Berger, H. (1976) Biochim. Biophys. Acta 426, 317-324

22 Dasseux, J.L., Faucon, J.F., Lafleur, M., Pézolet, M. and Dufourcq, J. (1984) Biochim. Biophys. Acta 775, 37-50

23 Van Deenen, L.L.M. and De Haas, G.H. (1964) Adv. Lipid Res. 2, 168-229

24 Smaal, E.B., Romijn, D., Geurts van Kessel, W.S.M., De Kruijff, B. and De Gier, J. (1985) J. Lipid Res. 26, 633-637

25 Fiske, C.H. and SubbaRow, Y. (1925) J. Biol. Chem. 66, 375-389

26 Rouser, G., Fleischer, S. and Yamamoto, A. (1975) Lipids. 5, 494-496

27 Hille, J.D.R., Donné-Op den Kelder, G.M., Sauve, P., De Haas, G.H. and Egmond, M.R. (1981) Biochemistry 20, 4068-4073

28 Uemura, A., Kimura, S. and Imanishi, Y. (1983) Biochim. Biophys. Acta 729, 28-34

29 Batenburg, A.M., Bougis, P.E., Rochat, H., Verkleij, A.J. and De Kruijff, B. (1985) Biochemistry 24, 7101-7110

30 Maulet, Y., Brodbeck, U. and Fulpius, B.W. (1982) Anal. Biochem. 127, 61-67

31 Habermann, E. and Jentsch, J. (1967) Hoppe-Seyler's Z. Physiol. Chem. 348, 37-50

32 Lübke, K., Matthes, S. and Kloss, G. (1971) Experienta 27, 765-767

33 Cavatorta, P., Spisni, A., Casali, E., Lindner, L., Masotti, L. and Urry, D.W. (1982) Biochim. Biophys. Acta 689, $113-120$

34 Bello, J., Bello, H.R. and Granados, E. (1982) Biochemistry 24, 461-465

35 Drake, A.F. and Hider, R.C. (1979) Biochim. Biophys. Acta $555,371-373$

36 Stoffel, W., Schreiber, C. and Scheefers, H. (1978) HoppeSeyler's Z. Physiol. Chem. 259, 923

37 Jain, M.K. and Maliwal, B.P. (1985) Biochim. Biophys. Acta $814,135-140$

38 Fung, B. and Stryer, L. (1978) Biochemistry 17, 5241-5248

39 Haigh, E.A., Thulborn, K.R. and Sawyer, W.H. (1979) Biochemistry 18, 3525-3532

40 De Kruijff, B., Cullis, P.R., Verkleij, A.J., Hope, M.J., Van Echteld, C.J.A., Taraschi, T.F., Van Hoogevest, P., Killian, J.A., Rietveld, A. and Van der Steen, A.T.M. (1985) in Progress in Protein-Lipid Interactions (Watts, A. and De Pont, J.J.H.H.M., eds.), pp. 89-142, Elsevier Biomedical Press, Amsterdam

41 Dufourcq, J., Dasseux, J.L. and Faucon, J.-F. (1984) Bacterial Protein Toxins 24, 127-138 
42 Knöppel, E., Eisenberg, D. and Wickner, W. (1979) Biochemistry $18,4177-4181$

43 Mollay, C. (1985) in The Enzymology of Post-translational Modifications of Proteins (Freedman, R. and Hawkins, M.C., eds.), Vol. 2, Academic Press, London
44 Inouye, M. (1978) Annu. Rev. Biochem. 47, 481-532

45 Von Heijne, G. and Blomberg, C. (1979) Eur. J. Biochem. 97, 175-181

46 Engelman, D.M. and Steitz, T.A. (1981) Cell 23, 411-422 